\title{
SUPERSOLUBLE GROUPS
}

\section{REINHOLD BAER}

We shall term a group $G$ supersoluble if every homomorphic image $H \neq 1$ of $G$ contains a cyclic normal subgroup different from 1 . Supersoluble groups with maximum condition, in particular finite supersoluble groups, have been investigated by various authors: Hirsch, Ore, Zappa and more recently Huppert and Wielandt. In the present note we want to establish the close connection between supersoluble groups and upper nilpotent groups; and we shall use these results to show that the maximum condition is satisfied by the subgroups of every finitely generated supersoluble group. Finally we give a characterization of the groups with maximum condition containing an upper nilpotent subgroup of finite index, since finitely generated supersoluble groups belong in this class.

Notations. $Z(G)$ is the center of $G$ and $[G, G]$ is the commutator subgroup of $G$. An element is said to be of order 0 if it generates an infinite cyclic group.

1. We begin with a derivation of the elementary properties of supersoluble groups in a form convenient for our use.

Definition. The group $G$ is supersoluble if every homomorphic image $H \neq 1$ of $G$ contains a cyclic normal subgroup different from 1 .

Of the various similar properties which are equivalent to supersolubility in case $G$ is finite two may be mentioned:

(a) every homomorphic image $H \neq 1$ contains an abelian normal subgroup of rank 1 ;

(b) if $N \neq 1$ is a normal subgroup, then there exists a normal subgroup $M$ such that $M<N$ and $N / M$ is cyclic (or only abelian of rank 1).

Some of the properties of supersoluble groups which we are going to derive are also true of groups with property (a). One may prove that supersoluble groups satisfy always some weak form of (b), and will have certainly property (b) if the maximum condition is satisfied by the normal subgroups of $G$. On the other hand it is easy to see that free groups have property (b), and so one cannot expect that a theory of groups with property (b) will prove very satisfactory.

LemMa 1. Subgroups and homomorphic images of supersoluble groups are supersoluble.

Received by the editors April 27, 1954. 
Proof. If $N$ is a normal subgroup of the subgroup $S$ of the supersoluble group $G$, and if $N<S$, then there exists among the normal subgroups $X$ of $G$ which satisfy $X \cap S \leqq N$ a maximal one, say $M$. Since $M \cap S \leqq N<S$, we have $M<G$. Because of the supersolubility of $G$ there exists a normal subgroup $V$ of $G$ such that $M<V$ and $V / M$ is cyclic. Because of the maximality of $M$ we have $V \cap S \$ N$ so that $N(V \cap S) / N$ is a normal subgroup different from 1 of $S / N$. Since

$$
\begin{aligned}
N(V \cap S) / N & \simeq(V \cap S) /(V \cap N) \\
& \simeq[(V \cap S) /(M \cap S)] /[(V \cap N) /(M \cap S)]
\end{aligned}
$$

and since

$$
(V \cap S) /(M \cap S) \simeq M(V \cap S) / M \leqq V / M,
$$

we see that $N(V \cap S) / N$ is cyclic as a homomorphic image of a subgroup of the cyclic group $V / M$. Hence the subgroup $S$ of $G$ is supersoluble. The supersolubility of homomorphic images of supersoluble groups is quite obvious.

REMARK 1. Consider a group theoretical property $\phi$ and assume that subgroups and homomorphic images of $\phi$-groups are $\phi$-groups. Term a group $G \phi$-soluble if every homomorphic image $H \neq 1$ of $G$ contains a normal $\phi$-subgroup different from 1 . Then the argument used in the proof of Lemma 1 shows that subgroups and homomorphic images of $\phi$-soluble groups are $\phi$-soluble.

LeMma 2. The following properties of the group $G$ are equivalent.

(i) If $N$ is a characteristic subgroup of $G$ and $N<G$, then $G / N$ contains a cyclic normal subgroup different from 1.

(ii) $G$ is supersoluble.

(iii) If $M$ and $N$ are normal subgroups of $G$, and if $M<N$, then there exists a normal subgroup $R$ of $G$ such that $M<R \leqq N$ and $R / M$ is cyclic.

Proof. It is clear that (iii) implies (ii) and that (ii) implies (i). Assume now the validity of (i) and consider normal subgroups $M$ and $N$ of $G$ such that $M<N$. There exist normal subgroups $X$ of $G$ satisfying $M=N \cap X$, for instance $X=M$; and among these normal subgroups $X$ there exists a maximal one, say $M^{*}$. Next we form the product $P$ of all the characteristic subgroups of $G$ which are contained in $M^{*}$. It is clear that $P$ is a characteristic subgroup of $G$ and $P \leqq M^{*}$. Next we form the product $Q$ of all the normal subgroups $Y$ of $G$ such that $P \leqq Y$ and $Y / P$ is cyclic. It is clear that $Q$ too is a characteristic subgroup of $G$. From $M^{*} \cap N=M<N$ we deduce $M^{*}<G$. Hence $P \leqq M^{*}<G$ and property (i) imply the existence 
of a cyclic normal subgroup different from 1 of $G / P$. Consequently $P<Q$. Since $P$ is the product of all the characteristic subgroups of $G$ which are part of $M^{*}$, the characteristic subgroup $Q$ of $G$ is not part of $M^{*}$. By the definition of $Q$ this implies the existence of a normal subgroup $V$ of $G$ with the following properties:

$$
P \leqq V, \quad V / P \text { is cyclic, } \quad V \text { is not part of } M^{*} \text {. }
$$

Since $P$ is part of $M^{*}$ and $V$ is not, $P \neq V$ so that $V / P$ is a cyclic group different from 1. Since $V$ is not part of $M^{*}, M^{*}<M^{*} V$. Since $M^{*} V$ is a normal subgroup of $G$, we deduce from the maximality of $M^{*}$ that $M^{*} \cap N=M<M^{*} V \cap N \leqq N$. Furthermore we find that

$$
\begin{aligned}
\left(M^{*} V \cap N\right) / M & =\left(M^{*} V \cap N\right) /\left(M^{*} \cap N\right) \\
& \simeq M^{*}\left(M^{*} V \cap N\right) / M^{*}=\left(M^{*} V \cap M^{*} N\right) / M^{*} \\
& =M^{*}\left(M^{*} N \cap V\right) / M^{*} \\
& \simeq\left(M^{*} N \cap V\right) /\left(M^{*} \cap V\right) \\
& \simeq\left[\left(M^{*} N \cap V\right) / P\right] /\left[\left(M^{*} \cap V\right) / P\right]
\end{aligned}
$$

and that $\left(M^{*} N \cap V\right) / P \leqq V / P$. Hence $\left(M^{*} V \cap N\right) / M$ is cyclic as a homomorphic image of a subgroup of the cyclic group $V / P$. Thus (iii) is a consequence of (i); and this completes the proof.

REMARK 2. The remark appended to Lemma 1 applies mutatis mutandis here too.

LEMMA 3. If $H \neq 1$ is a homomorphic image of the supersoluble group $G$, then there exists a characteristic abelian subgroup different from 1 in $H$.

Proof. If $H$ possesses finite normal cyclic subgroups different from 1, then $H$ possesses normal subgroups of order a prime $p$; and the product $P$ of all the normal subgroups of order $p$ of $H$ is clearly an abelian characteristic subgroup different from 1 . If $H$ possesses infinite cyclic normal subgroups, then their product $Q$ is a characteristic subgroup different from 1. If $\{a\}$ and $\{b\}$ are infinite normal subgroups, then either $\{a\} \cap\{b\}=1$ in which case certainly $a b=b a$; or else there exist integers $i, j$ such that $a^{i}=b^{j} \neq 1$. Since $\{a\}$ is a normal subgroup of $H$, we have $b^{-1} a b=a^{e}$ with $e= \pm 1$. Hence

$$
a^{i}=b^{-1} a^{i} b=\left(b^{-1} a b\right)^{i}=a^{e i}
$$

so that $e=1$, and we have again $a b=b a$. Thus $Q$ is certainly abelian. But $G$ is supersoluble so that $H$ contains cyclic normal subgroups different from 1, and this completes the proof. 
2. The elements of finite order in a supersoluble group need not form a subgroup, as may be seen from the following simple

Example 1. Denote by $A$ an abelian group, and denote by $G$ the group obtained by adjoining to $A$ an element $b$, subject to the relations:

$$
b^{2}=1, \quad b a b=a^{-1} \quad \text { for every } a \text { in } A .
$$

One verifies that $G$ is supersoluble, that every element in the coset $A b$ is of order 2, and that $G$ is therefore generated by its elements of order 2. But $G$ may contain all sorts of elements according to our choice of $A$.

It will be seen later that this example describes almost the worst that can happen; see Lemma 6 and Corollary 1 below.

Lemma 4. If the finite set $F$ of elements of finite order in the supersoluble group $G$ generates an infinite subgroup $S$ of $G$, then $S$ is not a torsion group and some elements in $F$ are of even order.

Proof. It is a consequence of Lemma 1 that $S=\{F\}$ is supersoluble. Since $S$ is a finitely generated infinite group, there exists a normal subgroup $M$ of $S$ with the following two properties:

$S / M$ is infinite; and if $X$ is a normal subgroup of $S$ and $M<X$, then $S / X$ is finite.

For a proof of the existence of $M$ see Baer [2, p. 166, $\$ 1$, Lemma 1]. Since $S$ is supersoluble and $M<S$, there exists by Lemma 2 a normal subgroup $N$ of $S$ such that $M<N$ and $N / M$ is cyclic. $S / N$ is finite by our choice of $M$; and from the infinity of $S / M$ it follows that $N / M$ is an infinite cyclic group. Consequently $S$ is not a torsion group. If $Z(S / M)$ were not 1 , then $(S / M) / Z(S / M)$ would be finite because of our choice of $M$; and this would imply the finiteness of the commutator subgroup $M[S, S] / M$ of $S / M$; see Baer $[1$, p. 163, Zusatz]. But $S$ is generated by the finite set $F$ of elements of finite order; and so $(S / M) /(M[S, S] / M)$ is a finite abelian group implying the finiteness of $S / M$, an impossibility. Hence $Z(S / M)=1$. This implies in particular that the infinite cyclic normal subgroup $N / M$ of $S / M$ is not part of the center of $S / M$. The automorphism group of an infinite cyclic group has order 2 ; the centralizer $C / M$ of $N / M$ is consequently a normal subgroup of $S / M$ which contains $N / M$ and whose index $[S: C]=2$. It follows that $C$ contains every element in $F$ whose order is odd and that $C$ does not contain the whole of $F$. Consequently $F$ contains elements of even order, q.e.d.

Lemma 5. If $p$ is a prime, if $F$ is a finite set of elements of finite order in the supersoluble group $G$ such that, for every $f$ in $F$, every prime 
divisor of the order of $f$ is greater than $p$, then $S=\{F\}$ is finite and every prime divisor of the order of $S$ is greater than $p$.

REMARK. This result is well known; see, for instance, Ore [1]. We offer a proof for the convenience of the reader.

Proof. It is a consequence of Lemma 1 that $S=\{F\}$ is supersoluble; and $S$ is finite by Lemma 4 , since the order of every element in $F$ is odd. There exist certainly normal subgroups $X$ of $S$ such that every prime divisor of $[S: X]$ is greater than $p$, for instance $X=S$; and among these normal subgroups $X$ there exists a minimal one $N$, since $S$ is finite. To prove our lemma we have to show that $N=1$. If this were not true, then there would exist normal subgroups $Y$ of $S$ such that $Y<N$; and among these normal subgroups $Y$ there exists a maximal one $M$. Then $M<N$ and $N / M$ is a minimal normal subgroup of $S / M$. Since $S$ is supersoluble, we deduce from Lemma 2 that the minimal normal subgroup $N / M$ of $S / M$ is cyclic and that its order is a prime $q$. Since the order of $S / M$ is $[S: N][N: M]=[S: N] q$, since $N$ is minimal among the normal subgroups $X$ such that every prime divisor of $[S: X]$ is greater than $p$, we find that every prime divisor of $[S: N]$ is greater than $p$ and $q \leqq p$. Denote by $C / M$ the centralizer of $N / M$ in $S / M$. Then $C$ is a normal subgroup of $S$ and $S / C$ is essentially the same as a group of automorphisms of the normal subgroup $N / M$ of $S / M$. Since $N / M$ is cyclic of order $q$, we have firstly $N \leqq C$ so that $[S: C]$ is a divisor of $[S: N]$; and we note secondly that $[S: C]$ is a divisor of the order $q-1$ of the group of all automorphisms of the cyclic group $N / M$ of prime number order $q$. Since $q \leqq p$ is smaller than every prime divisor of $[S: N], q-1$ and $[S: N]$ are relatively prime. Hence $[S: C]=1$ so that $S=C$; and consequently $N / M$ is part of the center $Z(S / M)$. Since the order $q$ of $N / M$ is smaller than every prime divisor of $[S: N]$, order and index of the normal subgroup $N / M$ of $S / M$ are relatively prime so that we may apply Schur's Theorem; see, for instance, Zassenhaus $[1$, p. 125, Satz 25]. Since $N / M$ is part of the center of $S / M$, it follows now that $S / M$ is the direct product of $N / M$ and of a subgroup $H$. Since the order $q$ of $N / M$ and the order $[S: N]$ of $H$ are relatively prime, $H$ contains all the elements in $S / M$ whose orders are prime to $q$. If $f$ is in $F$, then the prime divisors of the order of $f$ are greater than $p$. Since $q \leqq p$, the orders of $f$ and $M f$ are prime to $q$. Hence every $M f$ belongs to $H$ so that $H<S / M=H$, the desired contradiction. Thus $N=1$; and we see that every prime divisor of the order of $S=\{F\}$ is greater than $p$.

DEFINITION. If $G$ is a group and $p$ is a prime, then $G(p)$ is the totality of elements $g$ in $G$ with the property: 
every prime divisor of the order of $g$ is greater than $p$.

Note that every $G(p)$ is a set of elements of finite order and that the orders of the elements in $G(p)$ are all odd.

Proposition 1. If $G$ is a supersoluble group and $p$ a prime, then $G(p)$ is a characteristic subgroup of $G$.

Proof. If $x$ and $y$ belong to $G(p)$, then it follows from Lemma 5 that $\{x, y\}$ is a finite subgroup of $G$ and that every prime divisor of the order of $\{x, y\}$ is greater than $p$. Hence every prime divisor of the order of $x y^{-1}$ is greater than $p$ so that $x y^{-1}$ belongs to $G(p)$. Consequently $G(p)$ is a subgroup of $G$ which clearly is characteristic.

3. In this section we are going to establish the rather close connections between supersolubility and upper nilpotency. We recall the

Definition. The group $G$ is upper nilpotent if $Z(H) \neq 1$ for every homomorphic image $H \neq 1$ of $G$.

Since every subgroup of the center is a normal subgroup, upper nilpotent groups are supersoluble, though the converse is clearly false.

Proposition 2. If $G$ is supersoluble, then $[G, G]$ is upper nilpotent.

This fact has been established by Zappa [1] under the additional hypothesis that the maximum condition be satisfied by the subgroups of $G$.

Proof. Denote by $U$ the upper hypercenter of $[G, G]$; for its definition and properties see Baer [2, pp. 176-177, §2, Proposition 2 and Notational Remark ]. $U$ is a characteristic upper nilpotent subgroup of $[G, G]$ such that $Z([G, G] / U)=1$. If our proposition were false, then $U<[G, G]$. Since $U$ is a characteristic subgroup of the characteristic subgroup $[G, G]$, it is a characteristic subgroup of the supersoluble group $G$. There exists, by Lemma 2, a normal subgroup $N$ of $G$ such that $U<N \leqq[G, G]$ and $N / U$ is cyclic. If $W / U$ is the centralizer of $N / U$ in $G / U$, then $W$ is a normal subgroup of $G$ and $G / W$ is essentially the same as a group of automorphisms of the cyclic normal subgroup $N / U$ of $G / U$. Hence $G / W$ is abelian so that $[G, G] \leqq W$. Consequently

$$
1<N / U \leqq Z([G, G] / U)=1,
$$

a contradiction which proves that $[G, G]=U$ is upper nilpotent.

REMARK. The method of proof can easily be used to prove that the intersection of all normal subgroups with finite abelian quotient group is upper nilpotent. 
Definition. If $G$ is a group and $p$ is an odd prime, then $G(p)^{*}$ is the intersection of all the subgroups $X$ of $G$ such that $[G, G] \leqq X$ and $[G: X]$ is a divisor of $p^{*}=\prod_{q \leqq p}(q-1)$.

It is clear that $G(p)^{*}$ is a characteristic subgroup of $G$, that $G / G(p)^{*}$ is an abelian torsion group, and that the order of every element in $G / G(p)^{*}$ is a divisor of $p^{*}$. The prime divisors of orders of elements in $G / G(p)^{*}$ are therefore smaller than $p$. If $p^{\prime}$ is the greatest prime which is smaller than the [odd] prime $p$, then the prime divisors of orders of elements in $G\left(p^{\prime}\right)$ are greater than $p^{\prime}$ and hence are at least $p$. The orders of elements in $G\left(p^{\prime}\right)$ and those in $G / G(p)^{*}$ are therefore finite and relatively prime. Consequently

$\left(^{*}\right) G(p) \leqq G\left(p^{\prime}\right) \leqq G(p)^{*}$ for every odd prime $p$ and its predecessor $p^{\prime}$.

We are now ready to show the existence of a torsion subgroup containing "most" of the elements of finite order.

Lemma 6. If $G$ is supersoluble, then the elements of finite order in $G(3)^{*}$ form a characteristic subgroup of $G$.

Proof. We note first that $G(3)^{*}=G^{*}$ is just the intersection of all the subgroups of index 2 [or 1 ] in $G$. Next we form the product $M$ of all the normal torsion subgroups of $G^{*}$. Then $M$ is a characteristic torsion subgroup of $G^{*}$ and hence of $G, H=G / M$ is supersoluble, $H^{*}=G^{*} / M$ is the intersection of all the subgroups of index 2 [or 1 ] in $H$, and 1 is the only normal torsion subgroup of $H^{*}$.

The elements of finite order in a normal abelian subgroup $A$ of $H^{*}$ form a characteristic subgroup $B$ of $A$. Clearly $B$ is a normal torsion subgroup of $H^{*}$ so that $B=1$. Every abelian normal subgroup of $H^{*}$ is therefore torsionfree.

Assume now by way of contradiction that $H^{*}$ is not torsionfree. Then the elements of finite order in $H^{*}$ generate a characteristic subgroup $W \neq 1$ of $H^{*}$ and $H$. The center $Z(W)$ of $W$ is a characteristic subgroup of $W, H^{*}$, and $H$, and consequently $Z(W)$ is [abelian and] torsionfree. Since $W$ is not torsionfree, $Z(W)<W$; and hence there exists, by Lemma 2 and the supersolubility of $H$, a normal subgroup $V$ of $H$ such that $Z(W)<V \leqq W \leqq H^{*}$ and such that $V / Z(W)$ is cyclic. If a group is cyclic modulo its center, then it is abelian. Hence $V$ is an abelian normal subgroup of $H^{*}$, and consequently $V$ is torsionfree.

Assume first that $V / Z(W)$ is finite. If $w$ is in $W$ and $v$ is in $V$, then there exists a positive integer $i$ such that $v^{i}$ belongs to $Z(W)$. Hence $v^{i}=w^{-1} v^{i} w=\left(w^{-1} v w\right)^{i}$. But $v$ and $w^{-1} v w$ both belong to the torsionfree abelian normal subgroup $V$. Hence $v=w^{-1} v w$ or $v w=w v$, and this 
implies $Z(W)<V \leqq Z(W)$, an impossibility which proves the infinity of $V / Z(W)$.

Since $V / Z(W)$ is an infinite cyclic normal subgroup of $H / Z(W)$, its group of automorphisms has order 2 and the centralizer of $V / Z(W)$ in $H / Z(W)$ has index 1 or 2 and contains therefore $H^{*} / Z(W)$. Consequently $V / Z(W) \leqq Z\left[H^{*} / Z(W)\right]$. Consider now an element $v$ in $V$ and an element $w$ of finite order $n$ in $W$. Clearly $v w \equiv w v$ modulo $Z(W)$ so that $z=v^{-1} w^{-1} v w$ belongs to $Z(W)$. Since $w$ is in $W$, we deduce successively that

$$
w^{-1} v w=v z, \quad w^{-i} v w^{i}=v z^{i}, \quad v=w^{-n} v w^{n}=v z^{n} \quad \text { or } \quad z^{n}=1 \text {, }
$$

and this implies $z=1$, since $Z(W)$ is torsionfree. Hence $v w=w v$. Thus $v$ commutes with every element of finite order in $W$. Since $W$ is generated by its elements of finite order, $v$ belongs to $Z(W)$; and we see that $Z(W)<V \leqq Z(W)$, a contradiction.

Thus we have arrived at a contradiction by assuming that $H^{*}$ is not torsionfree. Hence $H^{*}$ is torsionfree; and it follows that the torsion subgroup $M$ of $G^{*}$ contains every element of finite order in $G^{*}$. Hence $M$ is the totality of elements of finite order in $G^{*}=G(3)^{*}$, as we wanted to prove.

CoROLlaRY 1. If $G$ is a supersoluble group, then finitely many elements of finite order in $G(3)^{*}$ generate a finite subgroup.

This one deduces readily from Lemmas 6 and 4 .

Proposition 3. If $p$ is an odd prime and $G$ supersoluble, then $[G(p)$ is a characteristic subgroup of $G$ and $] G(p)^{*} / G(p)$ is upper nilpotent.

It will be convenient to treat first the following special case.

Proposition 3*. If $G$ is supersoluble and $G(3)^{*}$ is torsionfree, then $G(3) *$ is upper nilpotent.

Proof. Suppose first that $N$ is a normal subgroup of $G$, that $N<G(3)^{*}$, and that $G(3)^{*} / N$ is torsionfree. If we let $H=G / N$, then $H$ is supersoluble and $H^{*}=H(3)^{*}=G(3)^{*} / N$ is torsionfree and infinite. By Lemma 2, there exists a cyclic normal subgroup $S \neq 1$ of $H$ which is part of $H^{*}$. Since $H^{*}$ is torsionfree, $S$ is an infinite cyclic group. Thus the group of automorphisms of $S$ has order 2, and the centralizer of $S$ in $H$ has index 1 or 2. The centralizer of $S$ in $H$ contains therefore $H^{*}$ so that $S \leqq Z\left(H^{*}\right)$. This proves that $Z\left(H^{*}\right) \neq 1$. Assume now by way of contradiction that $H^{*} / Z\left(H^{*}\right)$ is not torsionfree. Since $Z\left(H^{*}\right)$ is a characteristic subgroup of $H$, since $H^{*} / Z\left(H^{*}\right)$ $=\left[H / Z\left(H^{*}\right)\right](3)^{*}$, and since $H / Z\left(H^{*}\right)$ is supersoluble, we may apply 
Lemma 6. The elements of finite order in $H^{*} / Z\left(H^{*}\right)$ form consequently a characteristic subgroup $W / Z\left(H^{*}\right)$. Hence $W$ is a characteristic subgroup of $H$ such that $Z\left(H^{*}\right)<W \leqq H^{*}$. By Lemma 2 there exists a normal subgroup $V$ of $H$ such that $Z\left(H^{*}\right)<V \leqq W \leqq H^{*}$ and such that $V / Z\left(H^{*}\right)$ is cyclic. Since $W / Z\left(H^{*}\right)$ is a torsion group, $V / Z\left(H^{*}\right)$ is a finite cyclic group. Since $Z\left(H^{*}\right)$ is part of the center of $V, V$ is an abelian subgroup of the torsionfree group $H^{*}$. Consider now an element $v$ in $V$ and an element $x$ in $H^{*}$. Since $V / Z\left(H^{*}\right)$ is finite, there exists a positive integer $i$ such that $v^{i}$ belongs to $Z\left(H^{*}\right)$. Hence $v^{i}=x^{-1} v^{i} x=\left(x^{-1} v x\right)^{i}$. But $v$ and $x^{-1} v x$ both belong to the abelian torsionfree normal subgroup $V$ of $H$. Hence $v=x^{-1} v x$ or $x v=v x$. Thus $Z\left(H^{*}\right)<V \leqq Z\left(H^{*}\right)$, an impossibility. Thus we have shown the following fact:

If $N$ is a normal subgroup of $G$, if $N<G(3)^{*}$, and $G(3)^{*} / N$ is torsionfree, then $Z\left[G(3)^{*} / N\right] \neq 1$ and $\left[G(3)^{*} / N\right] / Z\left[G(3)^{*} / N\right]$ is torsionfree.

On the basis of this fact one proves by an obvious [transfinite] induction that the upper central chain of $G(3)^{*}$ terminates in $G(3)$ * and that therefore $G(3)^{*}$ is upper nilpotent.

Proof of Proposition 3. If $p$ is an odd prime, then $G(p) \leqq G(p)^{*}$ $\leqq G(3)^{*}$. Let $H=G / G(p)$. Then $H$ is supersoluble

$$
H(3)^{*}=G(3)^{*} / G(p), \quad H(p)^{*}=G(p)^{*} / G(p), \quad \text { and } \quad H(p)=1 .
$$

It is a consequence of Lemma 6 that the elements of finite order in $H(3)^{*}$ form a characteristic subgroup $T$ of $H$. Then $H(3)^{*} / T$ is torsionfree, and an immediate application of Proposition $3 *$ shows that $H(3)^{*} / T$ is upper nilpotent. The subgroup $T H(p)^{*} / T$ of $H(3)^{*} / T$ is consequently upper nilpotent too, and this implies the upper nilpotence of the isomorphic group $H(p)^{*} /\left[H(p)^{*} \cap T\right]$. Clearly $R$ $=H(p)^{*} \cap T$ is a characteristic subgroup of $H$; as a matter of fact $R$ is the totality of elements of finite order in $H(p)^{*}$.

We want to prove that $R$ is an upper hypercentral subgroup of $H(p)^{*}$; for a definition of this concept see Baer [2, p. 173, §2, Definition 2]. To do this consider the upper hypercenter $U$ of $H(p)^{*}$; for its characterization see Baer [2, pp. 176-177, §2, Proposition 2 and Notational Remark]. We recall the two relevant properties of $U$ : (a) every normal subgroup of $H(p)^{*}$ which is part of $U$ is an upper hypercentral subgroup of $H(p)^{*}$; (b) $Z\left[H(p)^{*} / U\right]=1$.

If $R$ were not an upper hypercentral subgroup of $H(p)^{*}$, then, by property (a), $R$ would not be part of $U$. Hence $U<R U \leqq H(p)^{*}$. By Lemma 2 there exists a normal subgroup $S$ of $H$ such that $U<S$ $\leqq R U$ and $S / U$ is cyclic. Since $H=G / G(p)$, the orders of elements of 
finite order in $H$ are divisible only by primes not exceeding $p$. Since $S / U$ is a cyclic subgroup of $R U / U \simeq R /(R \cap U)$, and since $R$ is a torsion subgroup of $H, S / U$ is a finite cyclic group and the prime divisors of its order do not exceed $p$. Clearly $S / U$ contains a cyclic subgroup $P / U$ of order a prime $q \leqq p$. Since $P / U$ is a characteristic subgroup of the normal subgroup $S / U$ of $H / U, P$ is a normal subgroup of $H$. Denote by $C / U$ the centralizer of $P / U$ in $H / U$. Then $C$ is a normal subgroup of $H$ and $H / C$ is essentially the same as a group of automorphisms of $P / U$. The group of all automorphisms of $P / U$ is a cyclic group of order $q-1$. Hence $H / C$ is a cyclic group of an order dividing $q-1$. Recalling the definition of $H(p)^{*}$ and the fact that $q \leqq p$ we see that $H(p)^{*} \leqq C$. Consequently

$$
1<P / U \leqq Z\left[H(p)^{*} / U\right]=1 \text {, }
$$

by (b), and this is clearly impossible.

Thus we have shown that $H(p)^{*} /\left[H(p)^{*} \cap T\right]$ is upper nilpotent and that $H(p)^{*} \cap T$ is an upper hypercentral subgroup of $H(p)^{*}$. Consequently $H(p)^{*}$ is upper nilpotent; see Baer $[2$, p. 176, $\$ 2$, Proposition 2, (viii) ]. This completes the proof.

Corollary 2. If $G$ is supersoluble, and if $p^{\prime}<p$ are two consecutive primes, then $G\left(p^{\prime}\right) / G(p)$ is upper nilpotent.

This is an immediate consequence of $\left(^{*}\right)$ and Proposition 3.

CoROLlaRy 3. The supersoluble group $G$ contains an upper nilpotent characteristic subgroup of finite index if $G /[G, G]$ is of finite rank and there exists only a finite number of primes $p$ such that $G$ contains elements of order $p$.

We recall that the group $H$ is said to be of finite rank if there exists a positive integer $n$ such that every finitely generated subgroup of $H$ may be generated by $n$ elements.

It is easy to construct examples showing the indispensability of either hypothesis of Corollary 3.

Proof. There exists, by hypothesis, an odd prime $p$ such that $G$ is free of elements of prime number order $q$ greater than $p$. Thus $G(p)$ $=1$ and, by Proposition $3, G(p)^{*}$ is upper nilpotent. We have pointed out before that $G / G(p)^{*}$ is an abelian torsion group the orders of whose elements are divisors of the integer $p^{*}$. Since $G /[G, G]$ is, by hypothesis, of finite rank, its homomorphic image $G / G(p)^{*}$ is likewise of finite rank, and consequently $G / G(p)^{*}$ is finite so that $G(p)^{*}$ is an upper nilpotent characteristic subgroup of finite index.

4. Subgroups of finitely generated soluble groups need not be 
finitely generated, as may be seen from the following obvious

ExAmple 2. Denote by $F$ the [non-abelian] free group of rank $n>1$, and form the commutator subgroup of the commutator subgroup of $F$, namely $F^{\prime \prime}=[[F, F],[F, F]]$. Let $G=F / F^{\prime \prime}$. Then $G$ is finitely generated and its commutator subgroup $[G, G]$ is a free abelian group of countably infinite rank.

Leмma 7. The commutator subgroup $[G, G]$ of the finitely generated group $G$ is finitely generated, if there exists a normal subgroup $N$ of $G$ with the following properties:

(a) $N \leqq[G, G]$ and $[G, G] / N$ is finitely generated;

(b) every element in $N$ belongs to a finitely generated normal subgroup of $G$ which is part of $[G, G]$.

Proof. There exists a finite set $F$ of generators of $G$. Clearly the set of commutators $[x, y]=x^{-1} y^{-1} x y$ with $x$ and $y$ in $F$ is finite too. By (a) there exists therefore a finite set $E$ of elements in $[G, G]$ with the following two properties:

(1) every $[x, y]$ with $x, y$ in $F$ belongs to $E$;

(2) $[G, G]=N\{E\}$.

If $e$ is an element in $E$ and $f$ is an element in $F$, then the commutators $[e, f]$ and $\left[e, f^{-1}\right]$ belong to $[G, G]=N\{E\}$. Consequently there exist elements $n^{\prime}(e, f), n^{\prime \prime}(e, f)$ in $N$ such that $[e, f] n^{\prime}(e, f)$ and $\left[e, f^{-1}\right] n^{\prime \prime}(e, f)$ both belong to $\{E\}$. The finite set of elements $n^{\prime}(e, f)$, $n^{\prime \prime}(e, f)$ for $e$ in $E$ and $f$ in $F$ is contained, by (b), in a finitely generated normal subgroup $M$ of $G$ which is contained in $[G, G]$. Consequently $S=M\{E\}$ is a finitely generated subgroup of $[G, G]$. If $f$ is an element in $F$, then $M=f^{-1} M f$; and if $e$ is an element in $E$, then

$$
\begin{aligned}
& f^{-1} e f=e[e, f]=\left(e[e, f] n^{\prime}(e, f)\right) n^{\prime}(e, f)^{-1}, \\
& f e f^{-1}=e\left[e, f^{-1}\right]=\left(e\left[e, f^{-1}\right] n^{\prime \prime}(e, f)\right) n^{\prime \prime}(e, f)^{-1}
\end{aligned}
$$

both belong to $M\{E\}$. Hence $f^{-1} E f$ and $f E f^{-1}$ are both part of $S$ so that $f^{-1} S f \leqq S$ and $f S f^{-1} \leqq S$. Consequently $f^{-1} S f=S$ for every $f$ in $F$. Since $G=\{F\}$, it follows that $S$ is a normal subgroup of $G$. Since the normal subgroup $S$ of $G$ contains every commutator $[x, y]$ with $x$ and $y$ in the set $F$ of generators of $G$ [by (1)], the quotient group $G / S$ is abelian. Hence $S \leqq[G, G] \leqq S$ so that $[G, G]=S$ is finitely generated, as we wanted to show.

REMARK 3. The method of proof can be used to obtain much more general criteria than Lemma 7.

THEOREM 1. The maximum condition is satisfied by the subgroups of finitely generated supersoluble groups. 
In other words: Subgroups of finitely generated supersoluble groups are finitely generated.

REMARK. It may be seen from Example 2 that it is impossible to substitute for the hypothesis of supersolubility the weaker requirement of solubility.

Proof. Assume that $G$ is a finitely generated supersoluble group. Then $G / G(p)^{*}$ is a finitely generated abelian group the orders of whose elements are bounded [by the defining integer $p^{*}$ ]. Hence

(1) $G / G(p)$ * is finite for every prime $p$.

If a normal subgroup of a finitely generated group has finite index, then it is finitely generated too; see, for instance, Baer $[2$, p. 166, $\$ 1$, Finiteness Principle]. By (1) therefore

(2) $G(p)^{*}$ is finitely generated for every prime $p$.

We recall next that the maximum condition is satisfied by the subgroups of every finitely generated upper nilpotent group and that the elements of finite order in such a group form a finite subgroup; see Baer [2, p. 203, §8, Theorem and p. 207, §8, Corollary]. Combining this result with (2) and Proposition 3 we obtain the following results.

(3) If $p$ is an odd prime, then the maximum condition is satisfied by the subgroups of $G(p)^{*} / G(p)$ and the elements of finite order in $G(p) * / G(p)$ form a finite subgroup.

By Proposition 2, $[G, G]$ is upper nilpotent. The elements of finite order in $[G, G]$ form therefore a characteristic subgroup $N$ of $[G, G]$ and $G$; see Baer [2, p. 193, $\S 6$, Theorem 1, (v)]. Furthermore $N$ is, as an upper nilpotent torsion group, the direct product of its primary components $N_{p}$ where $N_{p}$ designates the totality of elements of order a power of $p$ in $N$; see Baer [2, p. 198, $\$ 6$, Corollary 1]. Clearly

$$
N_{p} \leqq[G, G] \leqq G(p)^{*} \text { and } G(p) \cap N_{p}=1 .
$$

Thus $N_{p}$ is isomorphic to a torsion subgroup of $G(p)^{*} / G(p)$; and now we deduce from (3) the finiteness of every $N_{p}$. Since every $N_{p}$ is a characteristic subgroup of the characteristic subgroup $N$ of $[G, G]$, every $N_{p}$ is a finite characteristic subgroup of $G$. Since $N$ is the direct product of the $N_{p}$, every element in $N$ is contained in a finite characteristic subgroup of $G$ which is part of $[G, G]$.

Since $G$ is finitely generated, $G /[G, G]$ is a finitely generated abelian group and the elements of finite order in $G /[G, G]$ form consequently a finite subgroup. If the prime number $r$ is greater than the order of the torsion subgroup of $G /[G, G]$, then the orders of the elements in $G(r)$ are prime to the orders of the elements of finite order in $G /[G, G]$. Consequently $G(r) \leqq[G, G]$. 
Combination of (1) and (3) shows that the maximum condition is satisfied by the subgroups of $G / G(r)$. Since $G(r)$ consists of elements of finite order in $[G, G]$, we have $G(r) \leqq N$; and consequently $G / N$ is a homomorphic image of $G / G(r)$. Thus every subgroup of $G / N$ is finitely generated so that in particular $[G, G] / N$ is finitely generated. Hence hypotheses (a) and (b) of Lemma 7 are satisfied by the normal subgroup $N$ of the finitely generated group $G$. Hence $[G, G]$ too is finitely generated. Since $[G, G]$ is upper nilpotent [by Proposition $2]$, the maximum condition is satisfied by the subgroups of $[G, G]$; see Baer [2, p. 203, §8, Theorem]. Since $G$ is finitely generated, the maximum condition is satisfied by the subgroups of $G /[G, G]$, and consequently the maximum condition is satisfied by the subgroups of $G$, as we wanted to show.

COROLLARY 4. If $G$ is a finitely generated supersoluble group, then the elements of odd order in $G$ form a finite subgroup of $G$ and there exists an upper nilpotent characteristic subgroup of finite index in $G$.

Proof. The elements of odd order in $G$ form the subgroup $G(3)$ of $G$, by Proposition 1 . By Theorem $1, G(3)$ is finitely generated; and hence $G(3)$ is finite by Lemma 4 . This proves our first contention. This implies in particular that there exists only a finite number of primes $p$ such that $G$ contains elements of order $p$. Since $G /[G, G]$ is finitely generated, $G /[G, G]$ is of finite rank. Hence, by Corollary 3 , there exists an upper nilpotent characteristic subgroup of finite index.

5. The results of the preceding section will now be utilized for a characterization of the groups with maximum condition possessing an upper nilpotent subgroup of finite index.

TheOREM 2. The following properties of the group $G$ are equivalent.

(i) The maximum condition is satisfied by the subgroups of $G$ and there exists an upper nilpotent subgroup of finite index in $G$.

(ii) $G$ is finitely generated and there exists a supersoluble subgroup of finite index in $G$.

(iii) (a) The maximum condition is satisfied by the upper nilpotent normal subgroups of $G$;

(b) If $N$ is an upper nilpotent normal subgroup of $G$, then the maximum condition is satisfied by the finite normal subgroups of $G / N$;

(c) if $H$ is an infinite homomorphic image of $G$, then there exists an element $h \neq 1$ in $H$ which possesses only a finite number of conjugates in $H$.

Proof. It is quite obvious that (i) implies (ii). Assume conversely 
the validity of (ii). Then there exists a supersoluble subgroup $S$ of finite index $[G: S]$. Since $S$ possesses only a finite number of conjugate subgroups in $G$ [as the normalizer of $S$ in $G$ contains $S$ and has therefore finite index too], their intersection is, by Poincaré's Theorem, a normal subgroup $N$ of finite index [ $G: N]$. Since $G$ is finitely generated and $G / N$ is finite, $N$ is finitely generated; see, for instance, Baer [2, p. 166, $\S 1$, Finiteness Principle]. Since $N$ is part of the supersoluble group $S, N$ is a finitely generated supersoluble group and consequently the maximum condition is satisfied by the subgroups of $N$ [Proposition 1 and Theorem 1] and there exists an upper nilpotent subgroup $U$ of finite index $[N: U]$ in $N$ [Corollary 4]. Clearly $[G: U]=[G: N][N: U]$ is finite; and the maximum condition is satisfied by the subgroups of $G$, since the same is true for $N$ and the finite group $G / N$. Hence (i) and (ii) are equivalent.

Assume again the validity of (i). Then conditions (iii.a) and (iii.b) are obvious consequences of the maximum condition in $G$. Consider next an infinite homomorphic image $H$ of $G$. By (i), $H$ contains an upper nilpotent subgroup $S$ of finite index $[H: S]$. Since $H$ is infinite and $[H: S]$ is finite, $S$ is an infinite upper nilpotent group. Hence $Z(S) \neq 1$. If $z \neq 1$ is an element in $Z(S)$, then its centralizer $C$ in $H$ contains $S$. Hence $[H: C]$ is finite and $z$ possesses only a finite number of conjugates in $H$. Hence (iii.c) is true too showing that (iii) is a consequence of (i).

Assume finally the validity of (iii). Denote by $\theta$ the set of all normal subgroups $X$ of $G$ with the following property:

$(\theta)$ There exists a normal subgroup $Y$ of $G$ such that $X \leqq Y, G / Y$ is finite and $X$ is an upper hypercentral subgroup of $Y$.

[We recall that the normal subgroup $N$ of the group $L$ is termed an upper hypercentral subgroup of $L$, if

$$
(N / M) \cap Z(L / M) \neq 1
$$

for every normal subgroup $M$ of $L$ satisfying $M<N$, and we note the obvious fact that upper hypercentral subgroups are upper nilpotent.]

It is clear that $\theta$ contains $X=1$. Since subgroups in $\theta$ are upper nilpotent normal subgroups of $G$, there exists, by (iii.a), among the subgroups in $\theta$ a maximal one, say $M$. Denote by $P / M$ the product of all the finite normal subgroups of $G / M$. Since $M$ is an upper nilpotent normal subgroup of $G$, the maximum condition is satisfied by the finite normal subgroups of $G / M$ [by (iii.b)]. Hence $P / M$ is the product of finitely many finite normal subgroups of $G / M$, and consequently $P / M$ is finite. If $T / P$ is a finite normal subgroup of $G / P$, 
then $T / M$ is a finite normal subgroup of $G / M$. By the definition of $P$ this implies $T / M \leqq P / M$; and thus we see that 1 is the only finite normal subgroup of $G / P$.

Assume now by way of contradiction that $P \neq G$. Then $G / P$ is infinite, since $G / P \neq 1$. There exists, by (iii.c), an element $h \neq 1$ in $G / P$ which possesses only a finite number of conjugates in $G / P$. Denote by $g$ some element in $G$ such that $h=P g$. Since $h \neq 1, g$ does not belong to $P$. Since $h$ possesses only a finite number of conjugates in $G / P$, and since $P / M$ is finite, $M g$ possesses only a finite number of conjugates in $G / M$.

Denote by $F$ the finite set of elements conjugate to $M g$ in $H=G / M$. The centralizer $C$ of $F$ in $H$ is identical with the centralizer of the normal subgroup $N=\{F\}$ of $H$. Hence $C$ is a normal subgroup of $H$ and $H / C$ is essentially the same as a group of permutations of the finite set $F$. Consequently $H / C$ is finite, and hence

$$
N /(N \cap C) \simeq N C / C \leqq H / C
$$

is finite too. If $N \cap C$ were part of $P / M$, then $N \cap C$ would be finite, and consequently $N$ would be finite. This would imply that $N$ is part of $P / M$ so that in particular $g$ would belong to $P$, an impossibility. Hence $N \cap C=D$ is a normal subgroup of $H$ which is not part of $P / M$. Since the centralizer $E$ of $D$ contains $C, D \leqq Z(E)$ and $[H: E]$ is finite.

We recall next that $M$ is a $\theta$-subgroup of $G$. Consequently there exists a normal subgroup $K$ of $G$ such that $M \leqq K, G / K$ is finite, and $M$ is an upper hypercentral subgroup of $K$. Since $K / M$ and $E$ are normal subgroups of finite index in $H=G / M$, their intersection $Y / M=(K / M) \cap E$ is a normal subgroup of finite index in $H$. Hence $Y$ is a normal subgroup of finite index in $G$. Denote by $X$ the uniquely determined normal subgroup of $G$ which contains $M$ and satisfies

$$
X / M=(K / M) \cap D=(K / M) \cap E \cap D=(Y / M) \cap D .
$$

Since $D$ is part of the center of $E, X / M$ is part of the center of $Y / M$. Since $M$ is an upper hypercentral subgroup of $K$, and since $M \leqq Y$ $\leqq K, M$ is an upper hypercentral subgroup of $Y$. Since $X / M$ is part of the center of $Y / M, X$ is an upper hypercentral subgroup of $Y$. Since $X$ and $Y$ are normal subgroups of $G$, and since $[G: Y]$ is finite, $X$ belongs to $\theta$. Since $M$ is maximal in $\theta$ and $M \leqq X$, it follows that $M=X$. Hence $1=D \cap(Y / M)$. Since $Y / M$ has finite index in $G / M$, $D$ is finite. Every finite normal subgroup of $G / M$ is part of $P / M$. Hence $D \leqq P / M$. But we have shown before that $D$ is not part of $P / M$. Thus we have been led to a contradiction which proves that 
$G=P$, and thus we see that the upper nilpotent normal subgroup $M$ of $G$ has finite index in $G$.

Denote by $\Sigma$ the set of all normal subgroups $X$ of $G$ with the following properties:

( $\Sigma) X \leqq M$ and the maximum condition is satisfied by the subgroups of $X$.

It is clear that $\Sigma$ is not vacuous, since $X=1$ belongs to $\Sigma$. Since subgroups of upper nilpotent groups are upper nilpotent, and since $M$ is an upper nilpotent normal subgroup of $G, \Sigma$ is a not vacuous set of upper nilpotent normal subgroups of $G$. By (iii.a), there exists among the subgroups in $\Sigma$ a maximal one, say $W$. Assume by way of contradiction that $W<M$. Since $M$ is upper nilpotent and $M / W$ $\neq 1, Z(M / W) \neq 1$. If $z \neq 1$ is in $Z(M / W)$, then the centralizer of $z$ in $G / W$ contains $M / W$. But $[G: M]$ is finite so that the index of the centralizer of $z$ in $G / W$ is finite too. Hence $z$ possesses only a finite number of conjugates in $G / W$; and these naturally all belong to the characteristic subgroup $Z(M / W)$ of the normal subgroup $M / W$ of $G / W$. Consequently there exists a finitely generated normal subgroup $V / W$ of $G / W$ such that $1<V / W \leqq Z(M / W)$. Since $V / W$ is a finitely generated abelian group, the maximum condition is satisfied by the subgroups of $V / W$. Since the maximum condition is satisfied by the subgroups of the group $W$ in $\theta$, the maximum condition is satisfied by the subgroups of $V$. Since $V$ is a normal subgroup of $G$, and since $V$ is part of $M, V$ belongs to $\theta$. Since $W$ is maximal in $\theta$ and $W<V$, we have arrived at a contradiction which proves that $M=W$ belongs to $\theta$. Thus the maximum condition is satisfied by the subgroups of $M$ and by the subgroups of the finite group $G / M$, and this implies the validity of the maximum condition in $G$. Hence (i) is a consequence of (iii), and this completes the proof.

REMARK 4. Suppose that the maximum condition is satisfied by the subgroups of $G$ and that there exists an upper nilpotent subgroup $S$ of finite index $[G: S]$. Finitely generated upper nilpotent groups are known to contain torsionfree subgroups of finite index; see, for instance, Baer [3, p. 307, $\S 1$, Zusatz 3]. Consequently there exists a torsionfree subgroup $T$ of finite index $[S: T]$. Since $[G: T]$ is finite and $G$ is finitely generated, there exists a characteristic subgroup $R$ of $G$ whose index $[G: R]$ is finite and which is part of $T$; see Baer $[3$, p. 331, $\S 5$, Folgerung 3$]$. Thus we have established the existence of a characteristic subgroup $R$ of finite index $[G: R]$ which is a torsionfree upper nilpotent group.

REMARK 5. If the maximum condition is satisfied by the subgroups of $G$, and if there exists a finite normal subgroup $N$ of $G$ with super- 
soluble quotient group $G / N$, then one verifies easily the validity of condition (iii) of Theorem 2. Thus there exists an upper nilpotent subgroup of finite index too. It is not difficult to construct examples which show that the converse is false.

It is quite obvious that conditions (iii.a) and (iii.b) of Theorem 2 are satisfied whenever the maximum condition is satisfied by the normal subgroups. Not so obvious is the fact that conditions (iii.a) and (iii.b) are satisfied by every group $G$ whose abelian subgroups are finitely generated, a fact that may be proven, for instance, using results of Malcew [1].

\section{BiBLIOGRAPHY}

REINHOLD BAER

1. Endlichkeitskriterien für Kommutatorgruppen, Math. Ann. vol. 124 (1952), pp. 161-177.

2. The hypercenter of a group, Acta Math. vol. 89 (1953) pp. 165-208.

3. Das Hyperzentrum einer Gruppe. III, Math. Zeit. vol. 59 (1953) pp. 299-338.

A. I. MaLCEW

1. Infinite soluble groups (in Russian), Mat. Sbornik vol. 28 (1951) pp. 567-588. O. ORE

1. Contributions to the theory of groups of finite order, Duke Math. J. vol. 5 (1939) pp. 431-460.

G. $Z_{\text {APPA }}$

1. Sui gruppi di Hirsch supersolubili, Rendiconti del Seminario Matematico della Università di Padova vol. 12 (1941) pp. 1-11, 62-80.

Hans Zassenhaus

1. Lehrbuch der Gruppentheorie. I, Leipzig-Berlin, 1937.

UNIVERSITY OF ILLINOIS 